\title{
Quantifying unmet prevention needs among MSM in Europe through a multi-site bio-behavioural survey
}

Massimo Mirandola ${ }^{1}$, Lorenzo Gios ${ }^{1}$, Nigel Sherriff², Ulrich Marcus 3 , Igor Toskin ${ }^{4}$, Magdalena Rosinska ${ }^{5}$, Susanne Schink ${ }^{3}$,

Sharon Kühlmann-Berenzon 6 , Barbara Suligoi ${ }^{7}$, Cinta Folch ${ }^{8}$, Christiane Nöstlinger ${ }^{9,10}$, Sonia Dias ${ }^{11}$, Danica Stanekova ${ }^{12}$, Irena

Klavs $^{13}$, Saulius Caplinskas ${ }^{14}$, Alexandru Rafila ${ }^{15}$, Carolina Marin ${ }^{16}$, Ivailo Alexiev ${ }^{17}$, Lev Zohrabyan ${ }^{18}$, Teymur Noori ${ }^{19}$, Cinthia

Menel-Lemos ${ }^{20}$, on behalf of the SIALON II Network ${ }^{21}$

1. Infectious Diseases Section, Department of Diagnostics and Public Health, University of Verona, Verona, Italy

2. School of Health Sciences, University of Brighton, Brighton, United Kingdom

3. Department of Infectious Disease Epidemiology, Robert Koch Institute, Berlin, Germany

4. Department of Reproductive Health and Research, World Health Organization, Geneva, Switzerland

5. Department of Epidemiology of Infectious Diseases and Surveillance, National Institute of Public Health - National Institute of Hygiene, Warsaw, Poland

6. Department of Public Health Analysis and Data Management, Public Health Agency of Sweden, Solna, Sweden

7. Centro Operativo AIDS, Dipartimento di Malattie Infettive, Parassitarie ed Immunomediate, Istituto Superiore di Sanità, Rome, Italy

8. Centre d'Estudis Epidemiològics sobre les Infeccions de Transmissió Sexual i Sida de Catalunya (CEEISCAT), Dept Salut, Generalitat de Catalunya / CIBER Epidemiologia y Salud Pública (CIBERESP), Barcelona, Spain

9. Department of Public Health, Institute of Tropical Medicine, Antwerp, Belgium

10. Faculty of Psychology, University of Vienna, Austria

11.11 Escola Nacional de Saúde Pública, Centro de Investigação em Saúde Pública \& GHTM, Universidade NOVA de Lisboa, Portugal

12. NRC for HIV/AIDS, Slovak Medical University, Bratislava, Slovak Republic

13. National Institute of Public Health, Ljubljana, Slovenia

14. Centre for Communicable Diseases and AIDS, Mykolas Romeris University, Vilnius, Lithuania

15. National Institute of Infectious Diseases Prof. Dr. Matei Bals, Bucharest, Romania

16. ACCEPT, Bucharest, Romania

17. National Reference Laboratory of HIV, National Center of Infectious and Parasitic Diseases, Sofia, Bulgaria

18. Regional Support Team Joint United Nations Programme on HIV/AIDS (UNAIDS), Moscow, Russia

19. European Centre for Disease Prevention and Control, Stockholm, Sweden

20. Consumers, Health, Agriculture and Food Executive Agency (Chafea), Luxembourg

21. Members of the the Sialon II Network have been acknowledged at the end of this article

Correspondence: Lorenzo Gios (gios.lorenzo@gmail.com)

Citation style for this article:

Mirandola Massimo, Gios Lorenzo, Sherriff Nigel, Marcus Ulrich, Toskin Igor, Rosinska Magdalena, Schink Susanne, Kühlmann-Berenzon Sharon, Suligoi Barbara, Folch Cinta, Nöstlinger Christiane, Dias Sonia, Stanekova Danica, Klavs Irena, Caplinskas Saulius, Rafila Alexandru, Marin Carolina, Alexiev Ivailo, Zohrabyan Lev, Noori Teymur, Menel-Lemos Cinthia, on behalf of the SIALON II Network. Quantifying unmet prevention needs among MSM in Europe through a multi-site bioLev, Noori Teymur, Menel-Lemos Cinthia, on behalf of the SIALON II Network. Quantifying unmet prevention needs among MS
behavioural survey. Euro Surveill. 2018;23(49):pii=1800097. https://doi.org/10.2807/1560-7917.ES.2018.23.49.1800097

Introduction: The HIV epidemic represents an important public health issue in Europe particularly among men who have sex with men (MSM). Global AIDS Monitoring indicators (GAM) have been widely and jointly promoted as a set of crucial standardised items to be adopted for monitoring and responding to the epidemic. Methods: The Sialon II study, implemented in 13 European cities (2013-14), was a complex multicentre integrated bio-behavioural cross-sectional survey targeted at MSM, with a concomitant collection of behavioural and biological (oral fluid or blood specimens) data. Rigorous sampling approaches for hard-to-reach populations were used (time-location sampling and respondent-driven sampling) and GAM indicators were calculated; sampling frames were adapted to allow weighted estimates of GAM indicators. Results: 4,901 MSM were enrolled. HIV prevalence estimates ranged from $2.4 \%$ in Stockholm to $18.0 \%$ in Bucharest. When exploring city-level correlations between GAM indicators, prevention campaigns significantly correlated with levels of condom use and level of HIV testing among MSM. Conclusion: The Sialon II project has made an important contribution to the monitoring and evaluation of the HIV epidemic across Europe, integrating the use of GAM indicators within a second generation HIV surveillance systems approach and in participatory collaboration with MSM communities. It influenced the harmonisation of European data collection procedures and indicators via GAM country reporting and contributed essential knowledge informing the development and implementation of strategic, evidence-based HIV prevention campaigns for MSM.

\section{Introduction}

The HIV epidemic represents an important public health issue in Europe, particularly among men who have sex with men (MSM). For example, across the entire European Region, one new HIV diagnosis case in every four is attributable to MSM. Moreover, in 15 
TABLE 1

Definitions for numerators and denominators

\begin{tabular}{|l|c|c|}
\hline GAM & Nominator & \multicolumn{1}{c|}{ Denominator } \\
\hline $\begin{array}{l}1.11 \text { (Prevention } \\
\text { programme) }\end{array}$ & $\begin{array}{c}\text { Number of MSM who replied 'yes' to both questions related to the } \\
\text { prevention programmes as per GAM guidelines (knowledge of HIV testing } \\
\text { services and condoms received in the last 12 months in the context of broad } \\
\text { prevention campaigns - outreach service) }\end{array}$ & $\begin{array}{c}\text { Total number of MSM who } \\
\text { participated in the survey }\end{array}$ \\
\hline 1.12 (Condom use) & $\begin{array}{c}\text { Number of MSM who reported that a condom was used the last time they } \\
\text { had anal sex }\end{array}$ & $\begin{array}{c}\text { Number of MSM who reported } \\
\text { having had anal sex with a male } \\
\text { partner in the last 6 months }\end{array}$ \\
\hline 13 (HIV testing) & $\begin{array}{c}\text { Number of MSM who reported having been tested for HIV during the last 12 } \\
\text { months and who knew their results }\end{array}$ & $\begin{array}{c}\text { Number of MSM included in the } \\
\text { sample }\end{array}$ \\
\hline 1.14 (HIV prevalence) & Number of MSM with a reactive HIV test (based on laboratory results) & $\begin{array}{c}\text { Number of MSM tested for HIV in } \\
\text { the context of the survey }\end{array}$ \\
\hline
\end{tabular}

GAM: Global AIDS Monitoring indicators; MSM: men who have sex with men.

EU/EEA countries (Austria, Croatia, Cyprus, Czech Republic, Germany, Greece, Hungary, Ireland, Malta, Netherlands, Poland, Slovakia, Slovenia, Spain and the United Kingdom), MSM accounted for roughly $50 \%$ of all new HIV cases [1,2]. According to the European Centre for Disease Prevention and Control (ECDC) and the World Health Organization (WHO), the 2014 HIV prevalence among MSM aged 25 years or younger is $2.9 \%$, while for MSM older than 25 years, it is estimated to be $7.7 \%$. Thirty-four percent of HIV cases attributed to sex between men are usually diagnosed before the age of $30[1,3]$.

While over the last few years there has been a general increase in new HIV infections among MSM in the EU/ EEA, among young MSM the increase has been particularly noticeable [4]. Indeed, the number of MSM aged 20-24 years newly diagnosed with HIV almost doubled between 2004 and 2013, while in MSM aged 30-39 years old there seems to be a relative stabilisation of new cases [1]. To reverse these trends, there is a need for strategic, large-scale comprehensive and complementary prevention measures such as increased HIV and Sexually Transmitted Infections (STIs) testing, condom promotion, access to pre-exposure prophylaxis (PrEP) and early treatment initiations [2].

The picture is arguably even more problematic in some Eastern European and Central Asian countries where, although the HIV epidemic among this population is often similar, the existence of relatively more stigmatising environment(s) are probably less conducive to the reporting of data that could potentially deepen the understanding of mechanisms of HIV transmission among MSM [5].

In order to target prevention strategies effectively and to monitor their impact at a local/regional and country level, a better understanding of the epidemiological patterns and identification of the most affected subpopulations, are key enabling factors in tackling the multifaceted HIV epidemic. In Europe this particularly relates to MSM and there is a clear need for a harmonised collection of reliable and comparable data on epidemiology and coverage of prevention measures in this population.

Consequently, international agencies (namely, the Joint United Nations Programme on HIV/AIDS (UNAIDS), WHO and ECDC) have called for countries to use robust surveillance and monitoring systems that adopt common and standardised indicators [6]. A key part of this international effort for harmonisation is the promotion and implementation of second generation HIV surveillance systems (SGSS), which collect and link biological and behavioural data [6]. The Global AIDS Monitoring indicators (GAM) are part of the SGSS and comprise a set of standardised items widely and jointly promoted by the WHO and UNAIDS $[7,8]$.

However, despite concerted efforts by these international agencies, the implementation of such a methodological approach is patchy and requires both adoption and strengthening across countries [8]. Although country reporting to the UNAIDS GAM has improved consistently over the years, to date there remains considerable variability in response rates [8].

A recent review of the GAM reporting process has highlighted a lack of data relating to key populations. For example, in 2012, GAM data on key populations were reported in approximately only $30 \%$ of cases [9].

A standardised set of GAM indicators is crucial given their role in providing specific data and information to monitor the implementation of the Sustainable Development Goals (SDG) and the UNAIDS 90-9090 strategy, recently endorsed by the European Commission (EC) Communication on 'Next steps for a sustainable European future' [10]. The EC has cofunded several projects in the area of HIV/AIDS, two of which have aimed specifically to implement a joint survey across different EU/EEA countries adopting the main principles of SGSS and the GAM approach as 
TABLE 2

Enrolment method, mean age, age group, by city, European Union cities $(\mathrm{n}=13)$

\begin{tabular}{|c|c|c|c|c|c|}
\hline \multirow{2}{*}{ City } & \multirow{2}{*}{ Recruitment type } & \multirow{2}{*}{ Mean age (range) } & \multicolumn{2}{|c|}{ Age group in years (GAM disaggregation) } & \multirow{2}{*}{ Total } \\
\hline & & & $<25$ & $25+$ & \\
\hline Barcelona & TLS & $37.2(19-79)$ & 42 & 360 & 402 \\
\hline Brighton & TLS & $35.1(18-74)$ & 67 & 344 & 411 \\
\hline Brussels & TLS & $34.9(18-68)$ & 50 & 341 & 391 \\
\hline Hamburg & TLS & $38.0(18-79)$ & 39 & 368 & 407 \\
\hline Lisbon & TLS & $37.9(19-76)$ & 35 & 373 & 408 \\
\hline Ljubljana & TLS & $30.5(18-73)$ & 121 & 273 & 394 \\
\hline Sofia & TLS & $29.6(18-58)$ & 115 & 296 & 411 \\
\hline Stockholm & TLS & $31.7(18-81)$ & 77 & 289 & 366 \\
\hline Warsaw & TLS & $28.8(18-71)$ & 92 & 314 & 406 \\
\hline Bratislava & RDS & $30.3(18-62)$ & 118 & 282 & 400 \\
\hline Bucharest & RDS & $30.8(19-58)$ & 47 & 134 & 181 \\
\hline Verona & RDS & $31.9(18-70)$ & 104 & 293 & 397 \\
\hline Vilnius & RDS & $30.7(19-59)$ & 83 & 239 & 322 \\
\hline
\end{tabular}

GAM: Global AIDS Monitoring indicators; RDS: Respondent-Driven Sampling; TLS: Time-Location.

Cities presented with white background: TLS survey. Cities presented with light blue background: RDS survey.

cornerstones. The two projects are the Sialon project [11] and the more recent Sialon II project $[12,13]$. In particular, in the Sialon II project a total of 13 countries with very different cultural and social environments with 30 institutions including public health institutions and non-governmental organisations (NGOs) were involved. The meaningful participation of LesbianGay-Bisexual-Trans-plus (LGBT +) communities in all participating countries has been key in designing and implementing the study.

The value of the Sialon II project lays in the sampling approach (Time-Location Sampling (TLS) and Respondent-Driven Sampling (RDS)) and the use of GAM indicators. The methodology adopted allowed the weighted estimation of GAM indicators, presented in this manuscript. To our knowledge, this is the first paper delivering weighted estimates for GAM indicators in a large number of European cities set within the framework of a SGSS specifically targeting MSM.

The objectives of this paper are to present weighted estimates of the GAM indicators among MSM based on the Sialon II bio-behavioural survey implemented in 13 European cities (2013-14) and to discuss the usefulness of these GAM indicators in monitoring the HIV epidemic and responses across EU countries.

\section{Methods}

Detailed descriptions of the study procedures and methods have been published elsewhere [12]. Here we present a short overview of the main methodological aspects.

\section{Study design}

The Sialon II study was a complex multi-centre integrated bio-behavioural cross-sectional survey with a concomitant collection of behavioural data and biological data (oral fluid or blood specimens).

\section{Setting}

The survey was implemented in 13 European cities. The decision to use TLS or RDS in each study site was based on preliminary formative research and organisational issues. TLS was adopted in nine cities: Brussels (Belgium), Sofia (Bulgaria), Hamburg (Germany), Warsaw (Poland), Lisbon (Portugal), Ljubljana (Slovenia), Barcelona (Spain), Stockholm (Sweden) and Brighton (United Kingdom (UK)). The setting for data collection included social and/or commercial venues and cruising settings preliminarily identified through formative research [14] and then selected randomly for data collection sampling calendars. RDS was used in four cities: Verona (Italy), Vilnius (Lithuania), Bucharest (Romania) and Bratislava (Slovakia). Regarding the latter, enrolment was based on the individuals' social network and for the data collection locally accredited healthcare facilities (e.g. a hospital) were used. Data collection for all sites took place from April 2013 to November 2014 .

\section{Sample size}

The sample size estimation was carried out based on the results from the former Sialon I project and other available studies [11]. Based on assumptions of HIV prevalence in the target population of at most $15 \%$, a precision of $5 \%$, a significance level of $95 \%$ and a design effect of 2.0 provided a random clustered sample size calculation of 392 MSM per study site. Taking into account the possibility of invalid samples, a final 
target of 408 MSM per city for TLS and 400 for RDS was planned.

\section{Participants}

Inclusion criteria were having had any kind of sex with another man during the previous year before enrolment, providing informed consent and agreeing to donate either an oral fluid (TLS) or blood specimen (RDS).

The exclusion criteria were being younger than the legal age of consent (18 years old) or having already participated in the study.

\section{Data sources/measurement}

\section{Behavioural data}

Behavioural data were collected through a pen-andpaper self-administered questionnaire. Core items were developed in line with the GAM indicators [7]. To allow for sampling weight calculations, additional items were included in the questionnaires on the venues attendance (TLS) or on network size (RDS).

\section{Biological data}

Biological specimens were obtained from participants of both study arms (TLS/RDS). In cities where TLS was used, specimens were tested for HIV antibodies using Genscreen HIV $1 / 2$ version 2, Bio-Rad (Marne la Roquette, France). A total IgG antibodies ELISA test Human IgG ELISA Kit 1x96, Quantitative/Immunology Consultants Laboratory was also used for oral fluid (OF) sample testing suitability and quality control. All HIVreactive samples were re-tested with Vironostika HIV $\mathrm{Ag} / \mathrm{Ab}$, Biomerieux (Marcy-l'Étoile, France). Samples reactive to the first ELISA HIV test, but negative to the second, were classified as negative.

In cities where RDS was used, blood specimens were collected and processed for serum extraction according to the respective national guidelines for safety and quality assurance. Serum samples were tested with a HIV fourth generation ELISA/CLIA screening test. A Western Blot test was used to confirm the positive cases.

\section{Variables}

The variables used for the present analysis included the GAM indicators suggested for MSM target population [15]. All proposed items included in the GAM guidelines for MSM were used in the survey questionnaire. Numerators and denominators were defined as seen in Table 1.

\section{Statistical methods}

The analysis was carried out according to the GAM indicator guidelines [15]. For all indicators, estimates were carried out with the following age disaggregation: $<25$ years old and $\geq 25$ years old. Age was calculated on the basis of the self-reported year of birth. Analyses were conducted using STATA Version 14.1 (StataCorp,
College Station, Texas, United States). To allow calculation of the sampling weights, a specific procedure was devised on the basis of previous publications and methodological guidelines $[12,16,17]$.

For the TLS survey, individual weights were assigned as the inverse of the product of the following: (i) the probability of the participant being at the sampled venue given he was at the sampled venue type (number of visits to sampled venue/number of visits to all types of venues); (ii) the length of the sampling time (out of all Venue-Day-Time units on the particular day) and (iii) the proportion of sampled individuals during the event in relation to the estimated number of visitors during the sampling event, a modification of the method proposed by Karon and Wejnert [17].

For the RDS survey, and in line with a RDS approach [18], weighted estimates were calculated using RDS Analyst (www.hpmrg.org), a suite of R commands developed by Handcock and colleagues (2015 RDS Analyst: Software for the Analysis of Respondent-Driven, Sampling Data, Version 0.52). Gile's Sequential Sampler approach was used for calculating the sampling weights. This approach is based on the inclusion probabilities of members of the sample which are based on reported network sizes [16]. This method is recommended when the sample is a significant fraction of the target population. Therefore, in order to use this method, population size estimates were carried out for each city. The calculation was based on the total number of inhabitants for the city area and the expected percentage of MSM (according to the consensus among the project's scientists and according to the scientific literature, given there is currently no comprehensive and precise agreement among experts on MSM population size estimations within the general population) $[19,20]$. All point estimates were reported with their respective sample size, 95\% Confidence Intervals $(\mathrm{Cl})$, and estimated design effect.

Ethics

All procedures adopted in the present study were in line with the 1964 Helsinki declaration and its amendments. Survey protocols were approved by the appropriate ethics committee in each participating city as well as by both the WHO Research Project Review Panel (RP2) and the WHO Research Ethics Review Committee (ERC). The name or any other identifier of the MSM enrolled in the study was not collected. All respondents were entitled to collect their test result at a nominated centre indicated to the participant during study enrolment. In case of a positive result, further testing, counselling, clinical follow up and ARV treatment were provided in line with the respective national guidelines.

\section{Results}

A total of 4,901 MSM were enrolled across the 13 participating cities. In TLS study sites, 3,596 participants were enrolled, while in RDS sites a total of 1,305 participants were enrolled. Participants enrolled per 
enrolment method/city, age mean, Min-Max, age group, are shown in Table 2. A detailed description of the sample is available in the Sialon II project report [13].

\section{GAM 1.11 (Prevention programmes)}

In eight of the nine cities where TLS was implemented, more than half of respondents answered positively to both questions (Table 3, GAM 1.11). The sole exception was Warsaw where $28.8 \%$ (95\% Cl: 23.3-34.3) of the participants had been reached by a prevention programme. Where RDS was implemented, less than half of the MSM answered yes to both the GAM questions, except for the older participants ( $\geq 25$ years) in Bucharest (50.7\%; 95\% Cl: 38.4-62.9) and young MSM ( 25 years) in Vilnius (58.3\%; 95\% Cl: 43.4-72.3).

The highest percentages of MSM reporting to be reached with an HIV prevention programme in the last 12 months were reported in Sofia and Hamburg (88.4\%; 95\% Cl: 82.9-94.1 and 81.4\%; 95\% Cl: 77.685.1 respectively). In Bratislava and Warsaw, the lowest proportions of MSM participating in the survey had been reached by an HIV prevention programme (22.6\%; $95 \% \mathrm{Cl}: 17.0-26.8$ and $28.8 \%$; $95 \% \mathrm{Cl}: 23.3-$ 34.3 , respectively).

In all cities (except Brighton, Hamburg and Lisbon), older participants ( $\geq 25$ years) had been reached more over the last 12 months than the younger participants ( 25 years), although differences between these age categories were small in most cities.

\section{GAM 1.12 (Condom use)}

Condom use according to the GAM definition, ranged from $45.2 \%$ (95\% Cl: $38.1-51.4$ ) in Bratislava to $69.6 \%$ in Lisbon (95\% Cl: 64.5-74.3) (Table 3). With the exception of a few sites (Bratislava, Bucharest, Lisbon, Verona and Warsaw) condom use was higher for the young MSM category ( 25 years). Among older MSM ( $\geq 25$ years), estimates of condom use varied between $46.7 \%$ in Bratislava (95\% Cl: $38.5-54.8$ ) to $72.0 \%$ in Lisbon (95\% Cl: 65.2-77.9). It should be noted that for some cities, the number of participants in the younger age group was low and therefore the precision of the estimates is reduced.

\section{GAM 1.13 (HIV testing)}

The level of HIV testing as per GAM guidelines is reported in Table 3. The highest proportions of participants (total) reporting having received an HIV test within the last 12 months and who also knew the result of that test, were reported in Brussels $(68.1 \% ; 95 \% \mathrm{Cl}$ : 56.4-79.8) and Barcelona (63.0\%; 95\% Cl: 52.6-73.4). In Lisbon only $21.1 \%$ ( $95 \% \mathrm{Cl}: 12.5-29.8$ ) of younger men reported a HIV test with the collection of the test result in the last year, which represented the lowest levels in the study. The second lowest estimate after Lisbon was found in Bratislava with $29.0 \%$ (95\% Cl: 19.1-39.0) followed by Brighton 36.1\% (95\% Cl: 22.7-49.6) and Bucharest 39.0 (95\% Cl: 21.8-56.2). In several cities there were significant differences between age groups with regard to receiving an HIV test within the last 12 months and knowing the results. Participants from Brussels, Lisbon, and Warsaw reported differences greater than $20 \%$ for the two age groups.

\section{GAM 1.14 (HIV prevalence)}

HIV prevalence was calculated based on the oral fluidbased laboratory testing for TLS and from serum-based laboratory tests for RDS. HIV prevalence estimates varied by city with the lowest level reported in Stockholm $(2.4 \% ; 95 \% \mathrm{Cl}: 1.1-5.2)$ and the highest level in Bucharest (18.0\%; 95\% Cl: 9.1-27.0) (Table 3). Five cities had an HIV prevalence between 10-20\% (Brussels, Barcelona, Lisbon, Brighton, Bucharest), three cities between $5-10 \%$ (Hamburg, Warsaw, Verona), and five cities below $5 \%$ (Stockholm, Vilnius, Ljubljana, Bratislava and Sofia).

City-level correlations between GAM indicators When exploring city-level correlations between GAM indicators (Figure) a significant correlation was found between prevention programmes indicator (defined as condom availability and testing site knowledge) and HIV testing (correlation coefficient $0.52, p$-value $=0.006$ ). In addition, a correlation was found between preventive programmes indicator and condom use (correlation coefficient $0.45, p$-value $=0.022$ ). Data suggest that the higher the prevention programmes indicator the higher the level of condom use and testing among MSM, in both age groups.

\section{Discussion}

HIV remains a public health priority in the EU and the data from the Sialon II study has made an important contribution to the monitoring and evaluation of the HIV epidemic across Europe, by integrating the use of GAM indicators within a second generation HIV surveillance systems approach and in participatory collaboration with MSM communities. It has influenced the harmonisation of European data collection procedures and indicators via GAM country reporting and contributed essential knowledge informing the development and implementation of strategic, evidence-based HIV prevention campaigns for MSM.

The percentage of MSM reached with prevention programmes (as measured by GAM 1.11) showed significant differences both between and within cities. Although the use of different sampling methods can partially explain some of the differences between RDS vs TLS cities, the comparison among cities surveyed with the same sampling methodology provides valid indications on MSM prevention needs. In Warsaw, the number of individuals reached with prevention programmes is generally low, particularly for younger MSM. With the exception of Brighton, Hamburg, Lisbon and Vilnius, older MSM seem to be reached more frequently compared with younger MSM. Cities surveyed using RDS present, in general, a lower number of MSM reached with prevention programmes, and with the exception of Vilnius (where a specific programme was 


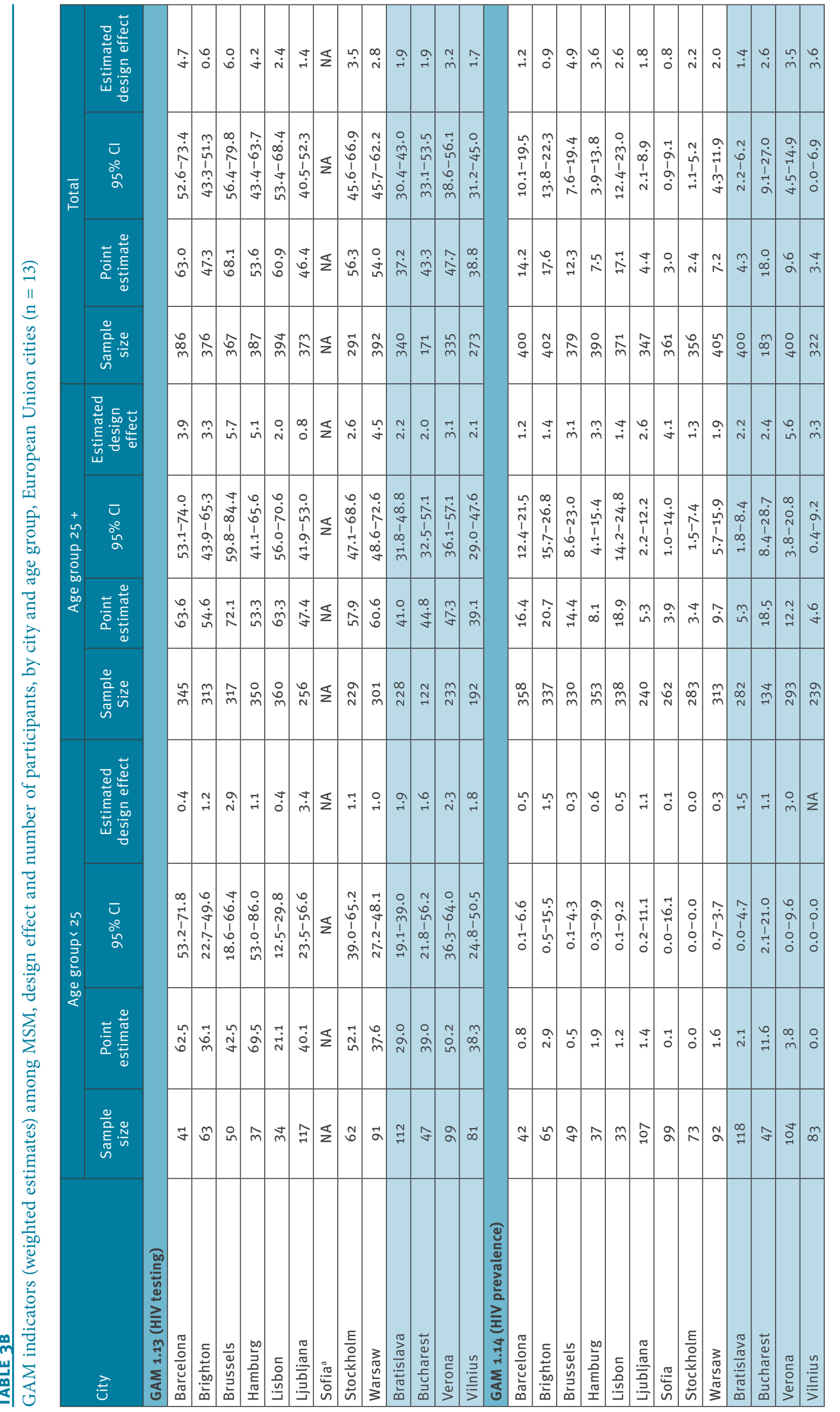




\section{FIGURE}

HIV testing (GAM 1.13) vs prevention programmes (GAM 1.11), European Union cities $(n=13)$

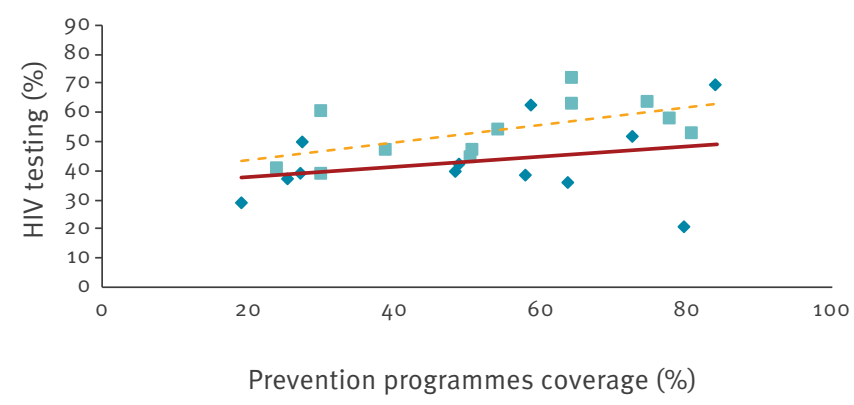

$$
\begin{aligned}
& \text { - HIV testing }<25 \quad \text { HIV testing } 25^{+} \\
& \text {- Linear (HIV testing <25) _ - Linear (HIV testing 25+) }
\end{aligned}
$$

run by an NGO before and during the data collection period), younger MSM showed the lowest level. These estimates are consistent with the literature and suggest the need for targeted prevention programmes tailored to locations and communities that can also accommodate for the needs of sub-populations, such as young MSM, MSM who are tourists and bisexuals [21,22].

Data focusing on the GAM 1.12 (Condom use) indicate that in the majority of the surveyed cities, condom use with any kind of partner was lower for younger men. However, the sample sizes for younger men were small for several cities and the association between age and condom use was not consistent across all study cities. In another publication based on the same Sialon-IIdataset, authors looked at condom use from another perspective e.g. any anal intercourse without a condom during the previous 6 months and not just from the last sexual encounter. The authors reported that almost half of the HIV-uninfected individuals reported condomless anal intercourse (CLAl). This was reported slightly more often by men living in Central European study cities and more frequently with steady partners compared with non-steady partners [23].

Based on the estimates of this GAM indicator there is a clear need to either increase condom use among younger men or to complement prevention strategies by providing meaningful access to other similarly effective HIV prevention tools such as HIV pre-exposure prophylaxis. Despite the fact that condom and lubricant distribution is often considered a simple and somewhat naïve approach to facilitate condom use, it is commonly acknowledged in the literature that fear of disapproval and discrimination by healthcare providers can deter gay, bisexual and other MSM from accessing mainstream health services [18]. Indeed, this limitation, could reduce access to free condom distribution, as well as low threshold HIV and STIs testing in healthcare settings [24].
A study by Marcus et al. [25] modelling the relationship between unprotected anal intercourse (UAI) and HIV disclosure with the same study dataset, found that among those respondents being aware of being HIV positive, condom use with steady partners was higher than among HIV negative men. However, condom use with non-steady partners was also lower. Men unaware of being infected with HIV reported the lowest condom use with non-steady partners $[23,25]$.

It is possible that a large number of MSM are unaware of their HIV status. It is estimated that in western and central Europe a noteworthy number of people at risk are not getting tested or can experience difficulties in being tested for HIV and STIs [2]. Based on surveillance data, ECDC estimates that a number of European countries may have a considerable proportion of late HIV diagnoses [2]. The testing behaviour as measured by the GAM 1.13 depicts a very different situation among the surveyed cities. In Barcelona, Hamburg, Sofia, Stockholm and Verona, approximately half of the participants (all ages) reported having received an HIV test within the last 12 months and knew the result of that test. In Brighton, Brussels and Warsaw more than half of older participants reported a known HIV test result within the last 12 months while among younger participants, it was below that level. In Lisbon only one in five younger men reported a known HIV test result in the last year which represented the lowest levels in the study.

In several cities there were differences between the two age groups with regards to receiving an HIV test within the last 12 months and knowing the results. For instance, participants from Brighton, Brussels, Lisbon, and Warsaw all reported differences of greater than $20 \%$ for the two age groups; however, considering the time span is limited to the last 12 months, the differences cannot be directly attributed to the age effect where, for example, older individuals may have had an increased opportunity to be tested over time. Furthermore, older participants were tested more frequently and within a shorter timeframe than the younger men, suggesting that increasing access to culturally sensitive HIV counselling, testing and antiretroviral therapy for MSM, found to have HIV, is an urgent health priority particularly for the younger generations. The current levels of HIV testing are insufficient to link gay, bisexual and other MSM with appropriate healthcare support shortly after acquiring HIV infection. Therefore, currently, testing frequencies can remain insufficient to effectively reduce the period of infectiousness of people who newly acquire HIV.

Alternative approaches such as the use of point of care tests (PoCTs) for HIV and STIs in low threshold community testing and LGBT venue-based testing, home collection testing, and HIV self-testing may represent effective approaches to increase diagnosis and linkage to care. 
The problem of late diagnosis reflects a lack of access to and uptake of HIV testing and counselling services in many countries [26]. A late diagnosis also means that a person has remained unaware of their HIV status for an indeterminate length of time, thus increasing the risk of transmitting the virus. The most recent surveillance data showed that despite significant efforts dedicated to the prevention and control of HIV, the rate of new HIV diagnoses has not substantially declined in the EU/EEA, but it has increased substantially over the last decade in the European Region.

Although HIV prevalence as an epidemic indicator is not a good parameter of the HIV infection spread dynamic, it can be helpful to provide an indirect picture of the epidemic history and patterns for some cities. HIV prevalence estimates (GAM 1.14), as measured by testing biological specimens (and not self-reported serological status), highlight critical levels of HIV infections across Europe among MSM communities despite valuable and concerted public health efforts [15].

Brussels, Barcelona, Lisbon, Brighton and Bucharest when considered globally, present relatively high HIV prevalences within the range of $10-20 \%$. That said, the HIV prevalence estimate for Bucharest is probably less reliable and interpretable compared with the other cities for two main reasons: the existence of an ostensible MSM sub-sample of injecting drug-users within the city sample, and the fact that the target number of MSM to be recruited was not reached (less than $50 \%$ of the estimated target sample was recruited). Compared to these relatively high HIV prevalences, more 'intermediate' levels of HIV prevalence were evident in four other participating cities (Hamburg, Warsaw, Verona, and Sofia) where it ranged from 5 to $10 \%$. Finally, in the cities of Stockholm, Vilnius, Ljubljana, and Bratislava, relatively lower HIV prevalences were observed with results below $5 \%$. Other smaller studies carried out in some of these cities (with different or similar sampling methods) have produced similar results $[11,27]$.

When exploring city-level correlations between GAM indicators, the data confirmed that prevention programmes correlate with both the level of condom use and of HIV testing among MSM, which might warrant additional efforts in implementing preventative actions. Despite numerous interventions targeting the behaviour, knowledge and attitudes of MSM, an increase of STIs and HIV diagnoses have been recently observed. Outbreaks of syphilis, lymphogranuloma venereum ( $L G V)$, hepatitis $C$ viral infection $(\mathrm{HCV})$ and other STIs have been reported in multiple European cities, possibility as a result of risky sexual behaviour and extensive sexual networking [26], but also may be due to an increase in active offering of HIV and STI's testing over the years.

A higher treatment coverage and higher percentage of HIV-positive MSM with undetectable viral load was attained in some Western European cities (Brussels,
Hamburg, Brighton and Verona), indicating that when the service provision is proactive and the treatment widely available, the link between testing and treating can effectively influence the HIV epidemic.

\section{Generalisability and limitations}

To our knowledge, this is the first paper presenting weighted estimates produced as a result of a standardised collection of GAM indicators for MSM in a large number of European cities adopting a common SGSS approach. The use of this approach (SGSS and GAM Indicators) and the active participation of key LGBT community stakeholders in the project's design and implementation, represent an asset that provides potentially usable data for both public health authorities and NGOs in each partner country.

The use of TLS and RDS methodologies within the context of a bio-behavioural survey using a participatory approach have allowed hidden and different subgroups of MSM to be reached; groups that are usually more difficult to access through surveillance studies [28]. Nevertheless, there are some limitations that should be taken into account when interpreting the results.

First, data can only be generalised to the particular MSM attending the gay venues in each study site (in the case of TLS survey) and only to those MSM socially linked to the gay community for each specific site (in case of the RDS survey). It has also been shown in other studies that these two sampling methods can result in different sample characteristics and the differences may persist even after applying weighting corrections [28].

Second, the generalisability of the findings may also be limited by contextual factors not measured in this survey (e.g. legislation and social norms) and an ecological fallacy cannot be excluded.

Third, an additional source of bias limiting generalisability relates to the self-reported behavioural data. This is of course an issue common to all surveys covering the self-reporting of sensitive information. However, the anonymity of the data collected and the self-administration of the questionnaire, with the careful design of the questionnaire items developed and validated in all cities with the involvement of the LGBT community, may well have reduced any social desirability effect [29]. It is also worth noting that no difficulties or limitations were reported by either respondents or data collectors with regards to the use of the GAM indicators (both TLS and RDS) in terms of interpretation and utility of the items and related indicators.

Fourth, the percentage of participants who reported ever having injected drugs ranged from $1.2 \%$ in Bratislava to $19.3 \%$ in Bucharest. In the latter, estimates might be difficult to be generalised due to this 
sub-population of injecting drug users (IDU)-MSM and to the fact that the target sample was not reached.

Fifth, the precision of the estimates were in some cases not optimal, particularly for the group of younger MSM, due to relatively small sample sizes (<50) and the potential for sampling bias in some cities.

Finally, an isolated limitation to the validity of the survey relates to Sofia (Bulgaria) where, due to an incorrect translation in the items questionnaire related to GAM 1.13, the indicator could not be estimated.

\section{Conclusions}

The Sialon II project and the data generated through its implementation represent a collaborative and scientifically robust contribution to the monitoring and evaluation of the HIV epidemic across Europe, integrating the use of GAM indicators within a SGSS approach with active community involvement. The data collected provide new evidence for appropriate planning of HIV prevention campaigns among MSM, clearly responding to the urgent need of concerted use of common indicators, with a particular focus on most at risk populations such as MSM [9]. The project has actively contributed to: (i) common procedures piloting (including most-advanced sampling methods, standards research algorithms, advanced laboratory diagnostics); (ii) harmonised data collection, involving experts from different institutions and with different backgrounds; and (iii) GAM country reporting, with specific reference to MSM.

\section{Acknowledgements}

Funding: This manuscript is based on data from the Sialon II project, co-funded under the Second Programme of Community Action in the field of Health (2008-2013) (Work Plan 2010). The sole responsibility lies with the authors of this manuscript and the Commission is not responsible for any use that may be made of the information contained therein.

The Sialon II Network: Massimo Mirandola, Lorenzo Gios, Stefano Benvenuti, Ruth Joanna Davis, Massimo Lunardi, Silvana Menichelli, Michele Breveglieri, Martina Furegato (Coordinamento Regionale per il Management e la Progettazione Europea, Azienda Ospedaliera Universitaria Integrata, Verona, Italy); Wim Vanden Berghe, Peter de Groot, Christiana Nöstlinger, Veronica van Wijk, Katrien Fransen, Tine Vermoesen, Michiel Vanackere (Institute of Tropical Medicine, Antwerp, Belgium); Fourat Benchikha, Sandra Van den Eynde, Boris Cruyssaert, Mark Sergeant, Karel Blondeel, Pieter Damen (Sensoa, Antwerp, Belgium); François Massoz, Erwin Carlier (Rainbowhouse Brussels, Belgium); Michael François, Stephen Karon (Ex Aequo, Belgium); Safia Soltani, Thierry Martin (Belgium); Alan De Bruyne (The Belgian Pride, Belgium); Françoise Bocken (Alias, Belgium); Myriam Dieleman (Observatoire du sida et des sexualités, Belgium); Ivailo Alexiev, Reneta Dimitrova, Anna Gancheva, Dobromira Bogeva, Maria Nikolova, Mariya Muhtarova, Todor Kantarjiev (National Center of Infectious and Parasitic Diseases, Sofia, Bulgaria); Viara Georgieva (National Center of Infectious and Parasitic Diseases, Sofia, Bulgaria; Ministry of Health, Sofia, Bulgaria); Emilia Naseva, Petar Tsintsarski, Hristo Taskov, Tonka Varleva (Program "Prevention and Control of HIV/AIDS", Ministry of Health, Sofia, Bulgaria); Elena Birindjieva, Aneliya Angelova, Manol Antonov (Association "Health without borders", Bulgaria); Ulrich Marcus, Susanne Barbara Schink, Sandra Dudareva-Vizule, Matthias an der Heiden, Sami Marzougui, Viviane Bremer, Andrea Kühne, Kerstin Schönerstedt-Zastrau, Ruth Zimmermann (Robert Koch Institute, Berlin, Germany); Andreas Wille (Institut für Hygiene und Umwelt, Hamburg, Germany); Kai Eckstein, Norman Buch, Philipp Moskophidis, Marc Grenz, Danilo Schmogro (Hein \& Fiete, Hamburg, Germany); Giuseppe Cornaglia, Antonella Zorzi, Elisabetta Tonolli, Giuliana Lo Cascio, Teresa Todeschini, Manuela Recchia, Lorella Pattini, Maria Rocca, Alessandra Bighignoli, Anita Galardi, Loredana Martini, Sandro Caffi, Pier Paolo Benetollo, Francesco Cobello, Chiara Bovom, Umberta Benvenuti (Azienda Ospedaliera Universitaria Integrata, Verona, Italy); Giulia Bisoffi, Oscar Bortolami, Laura Crestani (Unità Supporto alla Ricerca e Biostatistica, Azienda Ospedaliera Universitaria Integrata, Verona, Italy); Fabiano Comperini (Italy); Ercole Concia, Emanuela Lattuada, Massimiliano Lanzafame, Stefania Leonardi, Paola Del Bravo (Infectious Diseases Section, Department of Pathology, Verona University Hospital, Veneto Region, Verona, Italy); Maddalena Cordioli, Fabio Rigo, Emanuele Guardalben, Ivan Marchesoni (Università degli studi di Verona, Verona, Italy); Barbara Suligoi, Vincenza Regine, Lucia Pugliese (Centro Operativo AIDS, Istituto Superiore di Sanità, Rome, Italy); Saulius Caplinskas, Irma Caplinskiene, Rima Krupenkaite (Centre for Communicable Diseases and AIDS, Vilnius, Lithuania); Gediminas Sargelis, Arturas Rudomanskis ("Tolerant Youth Association", Vilnius, Lithuania); Sónia Dias, Ana Gama, Oriana Brás (Global Health and Tropical Medicine, Instituto de Higiene e Medicina Tropical, Universidade Nova de Lisboa, Portugal); João Piedade (Medical Microbiology Unit, Instituto de Higiene e Medicina Tropical, Lisbon, Portugal); Ricardo Fuertes, Nuno Pinto, João Brito, Júlio Esteves, Jesus Rojas, Fernando Ferreira, Miguel Rocha, Hugo Machado, Maria José Campos (CheckpointLX, Portugal); Luís Mendão (Grupo Português de Ativistas sobre Tratamentos de VIH/SIDA - Pedro Santos, Portugal); Magdalena Rosińska, Bożena Kucharczyk, Marta Niedźwiedzka-Stadnik, Łukasz Henszel, Andrzej Zieliński, Michat Czerwiński (National Institute of Public Health National Institute of Hygiene, NIPH-NIH, Warsaw, Poland); Michat Pawlęga, Ewelina Burdon, Małgorzata Gajdemska, Agnieszka Guściora, Nikodem Klasik, Katarzyna Rżanek, Michat Sawicki, Michat Tęcza (Lambda Warszawa, Warsaw, Poland); Mateusz Dębski, Anna Maciejewska, Izabela Pazdan (SKA Warsaw, Poland); Alexandru Rafila, Daniela Pitigoi, Adrian Abagiu (National Institute for Infectious Diseases Prof. Dr. Matei Bals, Bucharest, Romania); Carolina Marin, Ioana Panzariu, Alexandru Miroiu (ACCEPT Association, Bucharest, Romania); Madalina Popa, Monica Likker (National Institute for Infectious Diseases Prof. Dr. Matei Bals, Bucharest, Romania); Maria Georgescu, Galina Musat, Dan Cojocaru, Mihai Lixandru, Raluca Teodorescu (Romanian Anti-AIDS Association - ARAS, Bucharest, Romania); Danica Staneková, Monika Hábeková, Tatiana Drobková, Zuzana Chabadová, Soňa Wimmerova, Maria Mojzesová (Slovak Medical University, NRC for HIV/AIDS Prevention, Bratislava, Slovakia); Filip Kunč, Michal Skurák, Peter Bodnar, Katarína Horniaková, Mária Krahulcová, Jarmila Präsensová (Slovakia); Martin Smoleň, Peter Záhradník, Pavol Tibaj (NGO Dúhové srdce, Bratislava, Slovakia); Irena Klavs, Tanja Kustec, Claudia Adamič (National Institute of Public Health, Ljubljana, Slovenia); Mario Poljak, Robert Krošelj, Jana Mlakar (Institute of Microbiology and Immunology, Medical Faculty, University of Ljubljana, Ljubljana, Slovenia); Miran Šolinc (Association SKUC, Ljubljana, Slovenia); Cinta Folch, Laia Ferrer, Alexandra Montoliu, Jordi Casabona, Anna Esteve, Montserrat Galdon (Centre for Epidemiological Studies on HIV/STI in Catalonia CEEISCAT, Dept Salut, Generalitat de Catalunya, Barcelona, Spain); Victoria Gonzalez (Microbiology Service, Hospital Universitari Germans Trias i Pujol, Barcelona, Spain); Rafael Muñoz (StopSida, Barcelona, 
Spain); Maria Axelsson, Torsten Berglund, Sharon KühlmannBerenzon, Achilleas Tsoumanis, Inga Velicko (Public Health Agency of Sweden, Stockholm, Sweden); Nigel Sherriff, Christina Panton, Glynis Flood (School of Health Sciences, University of Brighton, Brighton, UK); Katrien Fransen, Tine Vermoesen (Aids Reference Laboratory, Institute of Tropical Medicine, Antwerp, Belgium); Ross Boseley, Marc Tweed (Terrence Higgins Trust, South, UK); Jonathon Roberts (Claude Nicol Centre, Royal Sussex County Hospital, Brighton, UK); Cinthia Menel Lemos (Executive Agency for Health and Consumers); Paolo Guglielmetti, Wolfgang Philipp, Matthias Schuppe (DG SANTE); Andrew Amato, Irina Dinca, Karin Haar, Anastasia Pharris, Teymur Noori (European Centre for Disease Prevention and Control ECDC); Igor Toskin, Armando Seuc, Natalie Maurer (Department of Reproductive Health \& Research of the World Health Organization, WHO); Lev Zohrabyan, Alexandrina lovita, Maddalena Campioni, Patrick Noack (Joint United Nations Programme on HIV/AIDS UNAIDS); Rosanna Peeling (London School of Hygiene and Tropical Medicine); Lisa Johnston (USA).

\section{Conflict of interest}

None declared.

\section{Authors' contributions}

MMI, LGI, NSH, UMA, ITO, MRO, SSC, SKB, BSU, CFO, CNO, SDI, DST, IKL, SCA, ARA, CMA, IAL, LZO, TNO, CML participated in the survey design and/or implementation. The first manuscript draft was jointly written by LGI and MMI. All authors (MMI, LGI, NSH, UMA, ITO, MRO, SSC, SKB, BSU, CFO, CNO, SDI, DST, IKL, SCA, ARA, CMA, IAL, LZO, TNO, CML) contributed writing to the following drafts. All authors read and approved the final manuscript. All authors fulfil the ICMJE authorship criteria.

\section{References}

1. European Centre for Disease prevention and Control (ECDC) ECDC SPECIAL REPORT. Thematic report: Men who have sex with men. Monitoring implementation of the Dublin Declaration on Partnership to Fight HIV/AIDS in Europe and Central Asia: 2014 progress report. Stockholm: ECDC; 2015 . Available from: https://ecdc.europa.eu/sites/portal/files/media/en/ publications/Publications/dublin-declaration-msm-2014.pdf

2. European Centre for Disease prevention and Control (ECDC). HIV and men who have sex with men. Monitoring implementation of the Dublin Declaration on Partnership to Fight HIV/AIDS in Europe and Central Asia: 2017 progress report. Stockholm: European Centre for Disease Prevention and Control; 2017. Available from: https://ecdc.europa.eu/sites/ portal/files/documents/HIV\%20and\%2omen\%20who\%20 have $\% 20$ sex $\%$ 2owith\%2omen.pd

3. European Centre for Disease Prevention and Control (ECDC). HIV/AIDS surveillance in Europe 2017 - 2016 data. Stockholm: ECDC; 2017. Available from: https://ecdc.europa.eu/en/ publications-data/hivaids-surveillance-europe-2017-2016-data

4. Pharris A, Quinten C, Tavoschi L, Spiteri G, Amato-Gauci AJECDC HIV/AIDS Surveillance Network. Trends in HIV surveillance data in the EU/EEA, 2005 to 2014: new HIV diagnoses still increasing in men who have sex with men. Euro Surveill. 2015;20(47):30071. https://doi.org/10.2807/15607917.ES.2015.20.47.30071 PMID: 26625124

5. The Joint United Nations programme on HIV and AIDS (UNAIDS). Global AIDS Update 2016. Geneva: UNAIDS; 2016. Available from: http://www.unaids.org/sites/default/files/media_asset/ global-AIDS-update-2016_en.pdf

6. UNAIDS, World Health Organization and Joint United Nations Programme on HIV/AIDS. Initiating Second Generation HIV Surveillance Systems: Practical Guidelines. Geneva: World Health Organization; 2002. Available from: http://www.who. int/hiv/pub/surveillance/en/isbn9291732192.pdf

7. The Joint United Nations programme on HIV and AIDS (UNAIDS). Global AIDS Monitoring 2018. Indicators for monitoring the 2016 United Nations Political Declaration on Ending AIDS.
Geneva: UNAIDS; 2017. Available from: http://www.unaids.org/ en/resources/documents/2018/Global-AIDS-Monitoring

8. Alfvén T, Erkkola T, Ghys PD, Padayachy J, Warner-Smith M, Rugg D, et al. Global AIDS Reporting-2001 to 2015: Lessons for Monitoring the Sustainable Development Goals. AIDS Behav. 2017;21(S1) Suppl 1;5-14. https://doi.org/10.1007/s10461-0161662-9 PMID: 28124296

9. Gall J, Sabin K, Frescura L, Sabin ML, Erkkola T, Toskin I. Global Trends of Monitoring and Data Collection on the HIV Response among Key Populations Since the 2001 UN Declaration of Commitment on HIV/AIDS. AIDS Behav. 2017;21(S1) Suppl 1;34-43. https://doi.org/10.1007/s10461-016-1618-o PMID: 27896550

10. European Commission (EC). Communication from the Commission to the European Parliament, the Council, the European Economic and Social Committee and the Committee of the Regions. Next steps for a sustainable European Future. European action for sustainability. COM(2016) 739 final. Strasbourg: EC; 22.11.2016. Available from: https://ec.europa. eu/europeaid/sites/devco/files/communication-next-stepssustainable-europe-20161122_en.pdf

11. Mirandola M, Folch Toda C, Krampac I, Nita I, Stanekova D, Stehlikova D, et al. HIV bio-behavioural survey among men who have sex with men in Barcelona, Bratislava, Bucharest, Ljubljana, Prague and Verona, 2008-2009. Euro Surveill. 2009;14(48):19427. https://doi.org/10.2807/ese.14.48.19427en PMID: 20003897

12. Gios L, Mirandola M, Toskin I, Marcus U, Dudareva-Vizule S, Sherriff N, et al. Bio-behavioural HIV and STI surveillance among men who have sex with men in Europe: the Sialon II protocols. BMC Public Health. 2016;16(1):212. https://doi. org/10.1186/s12889-016-2783-9 PMID: 26935752

13. The Sialon II Project. Report on a Bio-behavioural Survey among MSM in 13 European cities. ISBN 978-88-9876855-4 Cierre Grafica, 2016. Available from: http://sialon.eu/ data2/file/133 Sialon\%2oll Report\%200n\%20a\%20Biobehavioural\%20Survey $\% 20$ among $\% 20$ MSM\%20in $\% 2013 \% 20$ European\%2ocities.pdf

14. The Sialon II Network. Prevention Manual and Training. Formative Research Report. Prevention Training Manual. Deliverable of the SIALON II project (D7). 2012. Available from: http://sialon.eu/data2/file/133_347_SIALON_FR_report_final. pdf

15. The Joint United Nations programme on HIV and AIDS (UNAIDS). Joint United Nations Programme on HIV/AIDS. Global AIDS response progress reporting 2014: construction of core indicators for monitoring the 2011 UN political declaration on HIV/AIDS. Geneva: UNAIDS; 2014. Available from: http://www. unaids.org/sites/default/files/media_asset/GARPR_2014_ guidelines_en_o.pdf

16. Gile KJ. Improved inference for respondent-driven sampling data with application to HIV prevalence estimation. J Am Stat Assoc. 2011;106(493):135-46. https://doi.org/10.1198/ jasa.2011.apo9475

17. Karon JM, Wejnert C. Statistical methods for the analysis of time-location sampling data. J Urban Health. 2012;89(3):56586. https://doi.org/10.1007/S11524-012-9676-8 PMID: 22421885

18. Heckathorn DD. Respondent driven sampling: a new approach to study hidden populations. Soc Probl. 1997;44(2):174-99. https://doi.org/10.2307/3096941

19. Purcell DW, Johnson CH, Lansky A, Prejean J, Stein R, Denning $P$, et al. Estimating the population size of men who have sex with men in the United States to obtain HIV and syphilis rates. Open AIDS J. 2012;6:98-107. https://doi.org/10.2174/18746136 01206010098 PMID: 23049658

20. Marcus U, Hickson F, Weatherburn P, Schmidt AJEMIS Network. Estimating the size of the MSM populations for $38 \mathrm{EU}$ countries by calculating the survey-surveillance discrepancies (SSD) between self-reported new HIV diagnoses from EMIS and surveillance-reported HIV diagnoses among MSM in 2009. BMC Public Health. 2013;13:919. https://doi.org/10.1186/14712458-13-919 PMID: 24088198

21. Mirandola M, Gios L, Joanna Davis R, Furegato M, Breveglieri $M$, Folch C, et al. Socio-demographic factors predicting HIV test seeking behaviour among MSM in 6 EU cities. Eur J Public Health. 2017;27(2):313-8. PMID: 27615734

22. Mirandola M, Gios L, Sherriff N, Pachankis J, Toskin I, Ferrer L, et al. Socio-demographic Characteristics, Sexual and TestSeeking Behaviours Amongst Men Who have Sex with Both Men and Women: Results from a Bio-behavioural Survey in 13 European Cities. AIDS Behav. 2017;21(10):3013-25. https://doi. org/10.1007/s10461-017-1831-5 PMID: 28643241

23. Marcus U, Nöstlinger C, Rosińska M, Sherriff N, Gios L, Dias SF, et al. Behavioural and demographic correlates of undiagnosed HIV infection in a MSM sample recruited in 13 European cities. 
BMC Infect Dis. 2018;18(1):368. https://doi.org/10.1186/ S12879-018-3249-8 PMID: 30081839

24. Pachankis JE, Hatzenbuehler ML, Mirandola M, Weatherburn P, Berg RC, Marcus U, et al. The Geography of Sexual Orientation: Structural Stigma and Sexual Attraction, Behavior, and Identity Among Men Who Have Sex with Men Across 38 European Countries. Arch Sex Behav. 2017;46(5):1491-502. https://doi. org/10.1007/s10508-016-0819-y PMID: 27620320

25. Marcus U, Schink SB, Sherriff N, Jones AM, Gios L, Folch C, et al. HIV serostatus knowledge and serostatus disclosure, with the most recent anal intercourse partner in a European MSM sample recruited in 13 cities: results from the Sialon-I study. BMC Infect Dis. 2017;17(1):730. https://doi.org/10.1186/ S12879-017-2814-x PMID: 29178847

26. European Centre for Disease prevention and Control (ECDC). STI and HIV prevention in men who have sex with men in Europe. Stockholm: ECDC; 2013. Available from: https:// ecdc.europa.eu/sites/portal/files/media/en/publications/ Publications/STI-HIV-prevention-MSM-in-Europe-21-Feb-2013. pdf

27. Ferrer L, Furegato M, Foschia JP, Folch C, González V, Ramarli $D$, et al. Undiagnosed HIV infection in a population of MSM from six European cities: results from the Sialon project. Eur J Public Health. 2015;25(3):494-500. https://doi.org/10.1093/ eurpub/cku139 PMID: 25161202

28. Kendall C, Kerr LR, Gondim RC, Werneck GL, Macena RH, Pontes MK, et al. An empirical comparison of respondentdriven sampling, time location sampling, and snowball sampling for behavioral surveillance in men who have sex with men, Fortaleza, Brazil. AIDS Behav. 2008;12(4) Suppl;S97-104. https://doi.org/10.1007/S10461-008-9390-4 PMID: 18389357

29. Rosenman R, Tennekoon V, Hill LG. Measuring bias in selfreported data. Int J Behav Healthc Res. 2011;2(4):320-32. https://doi.org/10.1504/IJBHR.2011.043414 PMID: 25383095

\section{License and copyright}

This is an open-access article distributed under the terms of the Creative Commons Attribution (CC BY 4.0) Licence. You may share and adapt the material, but must give appropriate credit to the source, provide a link to the licence, and indicate if changes were made.

This article is copyright of the authors or their affiliated institutions, 2018. 